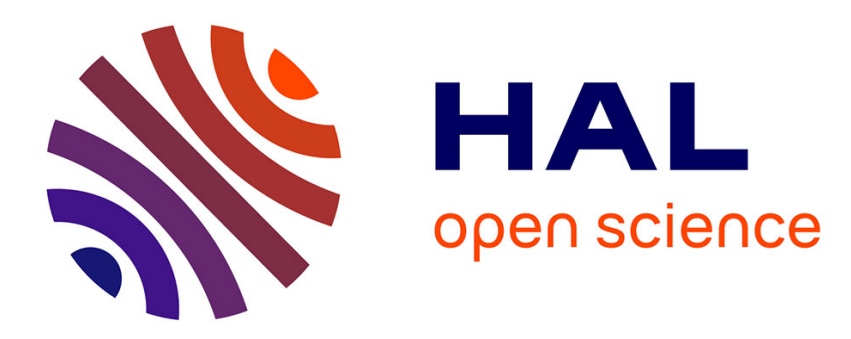

\title{
Controlling Stress Intensity Factor Histories with Digital Images
}

Xavier Fayolle, François Hild

\section{To cite this version:}

Xavier Fayolle, François Hild. Controlling Stress Intensity Factor Histories with Digital Images. Experimental Mechanics, 2014, 54 (2), pp.305-314. 10.1007/s11340-013-9790-x . hal-00947480

\section{HAL Id: hal-00947480 \\ https://hal.science/hal-00947480}

Submitted on 16 Feb 2014

HAL is a multi-disciplinary open access archive for the deposit and dissemination of scientific research documents, whether they are published or not. The documents may come from teaching and research institutions in France or abroad, or from public or private research centers.
L'archive ouverte pluridisciplinaire HAL, est destinée au dépôt et à la diffusion de documents scientifiques de niveau recherche, publiés ou non, émanant des établissements d'enseignement et de recherche français ou étrangers, des laboratoires publics ou privés. 


\title{
Controlling Stress Intensity Factor Histories with Digital
}

\section{Images}

\author{
Xavier Fayolle • François Hild ${ }^{\star}$
}

Received: date / Accepted: date

\begin{abstract}
It is shown that stress intensity factors (SIF) can be prescribed in mechanical tests by using digital image correlation. A priori resolutions are validated with a posteriori results. A cascade controller is implemented to prescribe SIF histories. A proof of concept is shown when testing a cracked elastomer. A tensile experiment is finally analyzed with a center cracked sheet made of commercially pure titanium. The linearity error is shown to be less than one order of magnitude higher for the closedloop system when compared to the SIF resolution. However, it is still small compared to the SIF ranges investigated herein.
\end{abstract}

Keywords Digital image correlation · fracture mechanics · full field measurements · measurement resolution

Laboratoire de Mécanique et Technologie (LMT-Cachan) ENS Cachan / CNRS / UPMC / PRES UniverSud Paris

61 Avenue du Président Wilson, F-94235 Cachan Cedex, France

${ }^{\star}$ Corresponding author: hild@lmt.ens-cachan.fr 
1 Introduction

The study of cracks is part of the theories describing the degradation of materials and failure of structures [1]. Crack initiation, propagation and arrest are concepts that are important to master to design and monitor actual structures such as airplanes, cars, trains, or power plants [2]. In many cases, elastic approaches are still used to assess the criticality of cracks. One way of mechanically characterizing cracks is to consider stress intensity factors (SIFs) that describe stress singularities in the vicinity of the crack tip, or equivalently, displacement discontinuities across crack faces [3]. It is the latter property that will be used hereafter in conjunction with full-field measurements.

Performing experiments on cracked samples or structures is a difficult task since crack propagation may become unstable. This is particularly true when load-controlled experiments are carried out since the SIF is generally increasing with the crack size. Conversely, when displacement controlled, the tests are usually easier to perform since crack propagation is likely to be stable. However, propagation may stop. The reason is that the parameters that control crack propagation are not necessarily the applied load or displacement, but crack opening displacements (CODs) [4,5], SIFs [6], energy release rates [7] or $J$-integral [8-10].

Except for CODs for which displacement transducers (so-called crack gauges) are used on large structures (e.g., bridges, buildings) or COD (clip) gauges on smaller ones, the other quantities are only indirectly evaluated when using point data, provided the crack length is known or monitored. Load shedding techniques whereby the applied load is gradually decreased as the crack propagates [11] have been proposed to keep the nonlinearities confined in the vicinity of the crack tip. However, the crack tip position needs to be determined to relate the applied load to the SIF level. Conversely, 
the sample geometry itself can be adapted to partially compensate for the load vs. SIF increase (e.g., edge-cracked tapered plate [12]). Yet another route consists of a linearly coupled load and displacement control loop that does not require the exact crack tip position to be determined [13]. One key parameter to tune is the so-called mixing ratio between displacement and remote load.

An alternative route is provided by full-field measurement techniques, in particular Digital Image Correlation (DIC [14,15]). Besides the qualitative aspect of detecting a crack, quantitative estimates of mechanically significant properties have been reported such as opening loads in fatigue experiments $[16,17]$ and/or SIFs [18-22] by least squares fit using closed-form solutions [3]. However, all these analyses were performed a posteriori and not during the experiment itself. Pictures have already been used to drive experiments. For instance, by tracking points, average strains are controlled [23]. This is also possible by resorting to DIC [24]. In both cases, experiments could be performed in the presence of localized strains. The advantage of DIC is that it is, by construction, a full-field measurement technique that was validated to analyze cracked media. In particular, its resolution may reach small values (typically 1 cpixel or even lower), which was shown to be compatible with the uncertainty level of strain gauges [24].

DIC is appealing when trying to control experiments, in particular when the input signals cannot be given by a strain gauge, an extensometer or a transducer (e.g., measuring a displacement or a load). DIC has already been used to control cracking experiments [25,26]. Either post-processed DIC measurements [25] or integrated DIC [26] have been used to evaluate the SIF history. In the present paper, the former approach is used to control experiments in which the crack does not propagate. The goal is to assess the resolution of the closed-loop system and to prove the feasibility 
on two very different materials (i.e., a very soft elastomer and a metallic material). To design the closed-loop software, the SIF resolution has to be assessed. This procedure is performed by first evaluating the displacement resolution of the implemented DIC code. Thereafter, it is possible to assess the resolution of SIFs. The performance of the controller is analyzed when an edge-cracked plate made of a soft material (i.e., elastomer) is tested. Last, experimental results are analyzed when an SIF history is prescribed on a center cracked sheet made of commercially pure titanium. In both cases, the a priori resolutions are compared with linearity errors for the open-loop and closed loop configurations.

\section{SIF Resolution}

When designing a closed-loop system, one key quantity is the resolution of the measuring device of the controlled variable (i.e., the SIF in the present case). To evaluate the SIF resolution, the displacement resolution is needed. It is based upon the analysis of the noise sensitivity of DIC [27,28]. The SIF resolution is then obtained by propagating the uncertainty induced by the least squares procedure to evaluate the mode I SIF from the measured displacements.

\subsection{Displacement Resolution}

In practice, the region of interest (ROI) is selected for the "SIF gauge." Within the ROI, smaller correlation windows or zones of interest (ZOIs) are chosen. For each ZOI in the reference image, its corresponding position is sought in subsequent pictures. The pattern-matching algorithm is based on cross-correlation. The cross-correlation is a function giving the maximum likelihood between two ZOIs shifted by a constant 
displacement. Its computation is performed via fast Fourier transforms [29-32]. The location of this maximum gives the most likely displacement between the ZOI in the reference and deformed configurations. For a sub-pixel estimate, a parabolic interpolation is performed around the maximum pixel value and the displacement corresponds to the maximum of the interpolated correlation function. Then the ZOI in the deformed configuration is shifted by an amount equal to the evaluated displacement using the shift/modulation property in Fourier space. Since interpolation errors occur, these three steps need to be iterated until convergence is reached. In the present case, since very small displacement increments are sought, a constant displacement assumption for each ZOI is deemed to be sufficient [24].

An estimate of the displacement resolution of the correlation algorithm is needed. The following procedure is applied to the actual picture of the experiment with the elastomeric material. The CCD digital camera used herein has a $1280 \times 1024$-pixel definition and a 12-bit dynamic range. Telecentric lenses are used to minimize the (spurious) effects of out-of-plane motion. To have independent estimates of the displacements in the following analyses, the shift $\delta$ between consecutive ZOIs is at least equal to the ZOI size $\ell$. The noise level of the camera is of the order of $\sigma=20$ gray levels. By following the derivations of Ref. [33], the corresponding standard displacement resolution is expressed as

$$
\sigma_{u}=\frac{\sqrt{2} \sigma p}{G_{f} \ell}
$$

where $G_{f}$ is the root mean square (RMS) value of the picture gradient (i.e., its mean contrast), $p$ the physical size of one pixel. 
These a priori results are compared with actual (i.e., a posteriori) measurements for which a set of 10 pictures is acquired for the same configuration. It was checked that the mean displacement was very small for all the analyzed cases. Figure 1 shows the standard displacement resolution $\sigma_{u}$ as a function of the ZOI size $\ell$; the larger the ZOI size, the smaller the resolution $\sigma_{u}$. However, if the ZOI size increases, the spatial resolution is degraded, i.e., less independent measurement points are obtained. The ZOI size also needs to be adapted to the speckle size (here of the order of $\approx 2$ pixels when characterized by an average correlation radius [34], i.e., sufficiently small with respect to $\ell$ ). Furthermore, as the ZOI size increases, so does the computation time. A good compromise for the present study is given by a ZOI size of 32 pixels for which a standard displacement resolution of the order of $2 \times 10^{-3}$ pixel is achieved.

Last, when interpolating the displacement resolution $\sigma_{u}$ by $A / \ell$, a value $A=7.4 \times$ $10^{-2}$ is found, to be compared to $\sqrt{2} \sigma / G_{f}=8.0 \times 10^{-2}$ (according to Equation (1) when $\sigma_{u}$ and $\ell$ are expressed in pixels). These two levels being close, the present results validate the a priori estimate of the standard displacement resolution $\sigma_{u}$. The low level of the displacement resolution is obtained thanks to a mean contrast $G_{f}$ of the order of 355 gray levels / pixel.

\subsection{SIF Resolution: A Priori Estimate}

The displacement field $\boldsymbol{u}$ in an infinite solid containing a semi-infinite crack along the $x$-axis $(x<0)$ is written as a combination of a double semi-infinite series [3]. In the following, only the dominant term associated with the mode I SIF is considered under a plane stress hypothesis (i.e., the considered samples have small thicknesses compared 
with the other dimensions)

$$
\begin{aligned}
& u_{x}=\frac{K_{I}}{E} \sqrt{\frac{r}{2 \pi}} \cos \frac{\theta}{2}[3-\nu-(1+\nu) \cos \theta] \\
& u_{y}=\frac{K_{I}}{E} \sqrt{\frac{r}{2 \pi}} \sin \frac{\theta}{2}[3-\nu-(1+\nu) \cos \theta]
\end{aligned}
$$

where $r, \theta$ denote the polar coordinates with respect to the crack tip, $x, y$ the cartesian coordinates with respect to the crack tip, E Young's modulus, and $\nu$ Poisson's ratio. This displacement field is rewritten as

$$
\boldsymbol{u}=K_{I} \boldsymbol{v}_{K}
$$

where $\boldsymbol{v}_{K}$ corresponds to the displacement field of a unitary mode I SIF.

Let us now assume that the displacement is known at a number of discrete points $\boldsymbol{x}_{n}$ (i.e., the center of each ZOI) with $n=1, \ldots, n_{x} n_{y}$ close to the crack tip, where $n_{x}, n_{y}$ denote the number of ZOIs in the $x$ and $y$ directions. The measured displacements are denoted by $\boldsymbol{u}_{m}$. The identification basis consists of fields associated with rigid body motions (i.e., translations $\boldsymbol{v}_{1}, \boldsymbol{v}_{2}$, rotation $\boldsymbol{v}_{3}\left(\boldsymbol{x}_{n}\right)$ ) and the mode I SIF field $\boldsymbol{v}_{4}\left(\boldsymbol{x}_{n}\right)=\boldsymbol{v}_{K}\left(\boldsymbol{x}_{n}\right)$. The corresponding amplitudes are determined by minimization of the quadratic cost function $\mathcal{T}$

$$
\mathcal{T}(\{\boldsymbol{a}\})=\sum_{n}\left\|\boldsymbol{u}_{m}\left(\boldsymbol{x}_{n}\right)-\sum_{i} a_{i} \boldsymbol{v}_{i}\left(\boldsymbol{x}_{n}\right)\right\|^{2}
$$

where $\{\boldsymbol{a}\}$ gathers the unknown translations $u_{0 x}, u_{0 y}$, rotation $\omega$, and SIF $K_{I}$. A linear system is solved

$$
[\boldsymbol{T}]\{\boldsymbol{a}\}=\{\boldsymbol{b}\}
$$

with

$$
T_{i j}=\sum_{n} \boldsymbol{v}_{i}\left(\boldsymbol{x}_{n}\right) \cdot \boldsymbol{v}_{j}\left(\boldsymbol{x}_{n}\right) \quad, \quad b_{i}=\sum_{n} \boldsymbol{v}_{i}\left(\boldsymbol{x}_{n}\right) \cdot \boldsymbol{u}_{m}\left(\boldsymbol{x}_{n}\right)
$$


It was mentioned that displacements are corrupted by measurement "noise," $\eta_{u}$, which is considered as white in the present case since each ZOI is independent of the other ones [34]. Consequently, the optimal SIF extractor is given by the least squares procedure [35]. Moreover, it was checked that the acquisition noise was a Gaussian distribution. It is of interest to investigate the effect of uncertainties on the resulting evaluation of $\{\boldsymbol{a}\}$. By considering the linear system to be solved for $\{\boldsymbol{a}\}$, it is observed that no systematic error (or bias) will result from the noise, and the covariance matrix $[\boldsymbol{C}]$ reads

$$
[\boldsymbol{C}]=[\boldsymbol{T}]^{-1} \sigma_{u}^{2}
$$

When the ROI is centered about the crack tip, the only off diagonal term of $[\boldsymbol{T}]$ is associated with $\boldsymbol{v}_{x} \cdot \boldsymbol{v}_{K}\left(\boldsymbol{x}_{n}\right)$. A first order estimate of the diagonal terms of the covariance matrix is given by (no index summation)

$$
C_{i i} \approx \sigma_{u}^{2} / T_{i i}
$$

which consists of neglecting any cross correlation between the various amplitudes (i.e., $C_{i j} \ll C_{i i}$, when $i \neq j$ ). Further, the following approximation is used to assess $C_{i i}$, which corresponds to the variance $\sigma_{i}^{2}$ of amplitude $a_{i}$, the other parameters being fixed,

$$
\frac{1}{n_{x} n_{y}} \sum_{n}\left\|\boldsymbol{v}_{i}\left(\boldsymbol{x}_{n}\right)\right\|^{2} \approx \frac{1}{\pi R_{\max }^{2}} \int_{0}^{R_{\max }} \int_{0}^{2 \pi}\left[v_{i x}^{2}(r, \theta)+v_{i y}^{2}(r, \theta)\right] r \mathrm{~d} r \mathrm{~d} \theta
$$

where $R_{\max }$ is determined by assuming a surface equivalence of the ROI

$$
\pi R_{\max }^{2}=L_{x} L_{y}
$$

where $L_{x}$ and $L_{y}$ denote the width and height of the ROI. The centers of the ZOIs correspond to the collocation points of the approximation of the previous integral. The standard SIF resolution $\sigma_{K}$ is normalized to become

$$
\frac{\sigma_{K} \sqrt{n_{x} n_{y} R_{\max }}}{E \sigma_{u}} \approx \sqrt{\frac{6 \pi}{19-10 \nu+3 \nu^{2}}}
$$


When the Poisson's ratio $\nu$ varies from -1 to 0.5 , the approximation to the dimensionless standard SIF resolution ranges from $\approx 0.77$ to $\approx 1.13$ (see Figure 2 ), i.e., of the order of unity ( since $\sqrt{6 \pi / 19} \approx 1$ ). Figure 2 shows that for small numbers of ZOIs (ranging from $n_{x}=n_{y}=2$ to 6 ), the proposed approximation is a good first order estimate. It can be shown that when the number of ZOIs becomes very large, the present approximations lead to an underestimation of the order of $20 \%$ of the standard SIF resolution.

Equation (11) also shows that the higher the Young's modulus $E$, the larger $\sigma_{K}$. Further, the larger $R_{\max }$, the lower $\sigma_{K}$. However, some care should be exercised since increasing $R_{\max }$ may induce a ROI that no longer belongs to the $K$-dominant region so that the chosen kinematic basis is no longer relevant. This can be checked by assessing the identification error

$$
\eta=\sqrt{\frac{\mathcal{T}(\{\boldsymbol{a}\})}{n_{x} n_{y}}}
$$

The closer $\eta$ to $\sigma_{u}$, the better the identification. It is also possible to decrease $\sigma_{K}$ by increasing $n_{x} n_{y}$, which is accompanied by an increase in computation time. A final way is to decrease the displacement resolution $\sigma_{u}$. This is achieved by considering larger ZOIs, again inducing a higher computation cost.

This last discussion shows that the SIF resolution $\sigma_{K}$ is the result of a trade-off between the resolution level and the computation time. This is particularly important for a closed-loop system in which the reference signal is obtained by the analysis of digital images. By using the results of the previous section, the SIF resolution becomes

$$
\frac{\sigma_{K} \sqrt{n_{x} n_{y} R_{\max }}}{E} \propto \frac{\sqrt{2} \sigma p}{G_{f} \ell}
$$

Equation (13) summarizes the different compromises that have to be considered when designing a controller with a DIC-based evaluation of SIFs. The left hand side term 
$\sigma_{K} \sqrt{n_{x} n_{y} R_{\max }} / E$ is related to the sought quantity itself, which is associated with analysis of $n_{x} n_{y}$ points in a ROI of characteristic radius $R_{\max }$ of a material with Young's modulus $E$. The second parameter, $\sqrt{2} \sigma p / G_{f} \ell$, is the displacement resolution, which is related to the analyzed texture (i.e., its mean contrast $G_{f}$ ) by a given camera (of acquisition noise $\sigma$ ) with a chosen magnification (i.e., $p$ ), and to the spatial resolution $\ell$. It is worth noting that the SIF resolution is valid when the crack tip is centered in the ROI. If this hypothesis is not satisfied, there are additional correlations that need to be accounted for. However, for the sake of simplicity, they are not considered in the following analyses.

Last, the fact that only the very first mode I displacement field associated with the presence of a crack is chosen is related to the limited number of measurement points, themselves linked to speed of the controller. This choice implicitly assumes that the ROI belongs to the K-dominant area and that a pure mode I loading is achieved. These hypotheses may be checked a posteriori with a more complete analysis considering more terms of Williams' series $[21,17,36]$ and more measurement points. Furthermore, the estimate of the stress intensity value may be biased as additional terms of Williams' series are needed to describe the measured displacement field. The level of the identification error is one means of checking whether the considered basis is sufficient when compared to the displacement resolution.

\section{Controlling SIF Histories via DIC}

The general setting of the controller is identical to the one that was used to prescribe strain histories to a sample [24], namely, a two-loop system is implemented to allow a slow outer loop to compute the current value of the SIF to be compared to the 
prescribed level. One cycle consists of an image acquisition, image saving (on the hard disk) and the evaluation time of the SIF through DIC calculations. It requires a total time of $2.4 \mathrm{~s}$. This is not a limitation per se as the use of GPUs may significantly reduce this time [37]. The aim here is to show the feasibility of SIF control, more than 'real time' issues. The inner loop consists of a displacement prescribed signal with a frequency of $20 \mathrm{~Hz}$ compatible with electromechanical (and servohydraulic) testing machines.

In the present application, the input signal is an SIF. In the following, the loading history is given by linear ramps loading and unloading the crack. To cancel out the steady state error between the input signal (i.e., a ramp) and the measured SIF (feedback), a double integral controller is implemented in a cascade system (Figure 3). However, a double integral controller, by itself, is unstable. A zero is added in the low frequency range to raise the phase by up to $45^{\circ}$, thereby yielding a sufficient phase margin (from the $180^{\circ}$ limit). Last, a unitary open loop gain is sought so that SIF signals are directly controlled.

Contrary to what was performed previously (i.e., using a servohydraulic testing machine [24]), an electromechanical testing machine is chosen. The machine manufacturer gives a rise time of $50 \mathrm{~ms}$ for $10 \mu \mathrm{m}$ displacement increments. To avoid accounting for the frequency response of the testing machine itself the computer process is delayed by $300-\mathrm{ms}$ hold time (i.e., only the steady state response is needed). The consequence is that the total cycle time of the outer loop is $2.7 \mathrm{~s}$ (rather than $2.4 \mathrm{~s}$ ). The testing machine is modeled as a stiff equation (i.e., no Laplace function). To check various aspects of the controller depicted in Figure 3 and to estimate the zero value of the controller Simulink ${ }^{\circledR}[38]$ is used. Figure 4 shows the error (E) and reference (R) signals. Apart from the changes of slope that induce high (unavoidable) errors, the system has 
a behavior that is consistent with what was expected (e.g., zero steady state error for ramps).

One important aspect of the controller is related to gain $G_{0}$ that links the prescribed SIF (outer loop) with the displacement of the testing machine crosshead (inner loop). To compute $G_{0}$, the total displacement associated with a sample of initial length $L_{0}$, cracked at its mid-height is needed. The edge crack size is denoted by $a$ and the width of the sample by $b$. The relative displacement $\Delta u_{p}$ of the two $L_{0}$-apart sections reads [39]

$$
\Delta u_{p}=L_{0} \frac{\Sigma}{E}\left(1+\frac{4 a v_{2}}{L_{0}}\right)
$$

where $\Sigma$ is the remote stress, and $v_{2}$ a dimensionless parameter dependent upon $a / b$.

The applied stress $\Sigma$ is related to the SIF $K_{I}$ by

$$
K_{I}=Y \Sigma \sqrt{\pi a}
$$

where $Y$ is a dimensionless parameter dependent upon $a / b$ [39]. By combining these two expressions, gain $G_{0}$ becomes

$$
G_{0}=\frac{\Delta u_{p}}{K_{I}}=\frac{L_{0}}{Y E \sqrt{\pi a}}\left(1+\frac{4 a v_{2}}{L_{0}}\right)
$$

All the parameters defining the SIF controller have now been introduced. The following section is a proof of concept of the implemented controller in a LabVIEW ${ }^{\mathrm{TM}}$ environment [40].

\section{Proof of Concept}

\subsection{Studied Material}

Being a feasibility study, the implemented controller is used to perform a test on a cracked elastomer. The displacement range was kept sufficiently low to avoid various 
non linear aspects associated with elastomers [41]. To evaluate the elastic parameters of the studied material, a tensile test on a dogbone sample has been carried out. Digital images were acquired during the test and the same DIC code was used to evaluate the mean longitudinal and transverse strains. The strain resolution $\sigma_{\epsilon}$ is determined by following the same type of derivation as for the SIF resolution. The only difference is given by the interpolating displacement field, which is linear (i.e., three unknowns per direction)

$$
\sigma_{\epsilon_{x x}}=\frac{\sigma_{u}}{L_{x} \sqrt{n_{x} n_{y}}} \sqrt{\frac{12\left(n_{x}-1\right)}{n_{x}+1}} \quad, \quad \sigma_{\epsilon_{y y}}=\frac{\sigma_{u}}{L_{y} \sqrt{n_{x} n_{y}}} \sqrt{\frac{12\left(n_{y}-1\right)}{n_{y}+1}}
$$

with $L_{x}=\delta\left(n_{x}-1\right)$ and $L_{y}=\delta\left(n_{y}-1\right)$. With the chosen parameters (i.e., $L_{y}>L_{x}=$ 350 pixels, $\ell=32$ pixels, and $n_{x}=n_{y}=4$ ) the strain resolution is less than $5 \times 10^{-6}$.

Figure 5 shows the stress/strain response of the material. As explained above, the stress and strain levels remain very low. By using a least squares fit of the measured data, a Young's modulus $E=7.6 \mathrm{MPa}$ and Poisson's ratio $\nu=0.4$ are obtained. Further, most of the fluctuations observed in Figure 5 are due to the load cell (whose resolution is of the order of $1 \mathrm{~N}$ or $0.02 \mathrm{MPa}$ ) and not the DIC code since the strain range is ten thousand times the corresponding resolution.

\subsection{A Posteriori Validation of Strain and SIF Resolutions}

With the experimental configuration, the strain resolution is less than $5 \times 10^{-6}$ and the SIF resolution is evaluated as $\sigma_{K}=2 \times 10^{-6} \mathrm{MPa} \sqrt{\mathrm{m}}$, since the physical size of one pixel is $p=53 \mu \mathrm{m}, R_{\max }=275$ pixels, and $\ell=32$ pixels. When performing the strain and SIF analyses on the same set of pictures as those used in the displacement resolution analysis, it is found that the strain resolution is of the order of $4 \times 10^{-6}$ and the SIF resolution $\sigma_{K}=2.1 \times 10^{-6} \mathrm{MPa} \sqrt{\mathrm{m}}$. These two values are very close to the $a$ 
priori estimates proposed herein. Consequently, the a priori displacement, strain and SIF resolutions are validated a posteriori.

\subsection{Open-Loop Response}

The response of the open-loop controller is shown in Figure 6. 137 pictures were acquired. Except for the changes of slope, the RMS difference with a piecewise linear response is of the order of $1.4 \times 10^{-5} \mathrm{MPa} \sqrt{\mathrm{m}}$. This value can be compared to the SIF resolution given above. There is a factor 7 between the two results.

To explain this difference, there are three types of cause to be listed. First, the resolution analysis performed herein does not account for sub-pixel interpolation errors [34]. In the present case, it is expected that they are non negligible since the displacement resolution is found to be very small. Second, the linearity error is also due to the crosshead displacement, which itself has its own resolution and errors. Last, the choice of only 4 displacement fields induces identification and model errors of the SIF level. It is expected that the model error be very small because of the low level of SIF. Conversely, the identification error is expected to increase with the SIF level. This trend was experimentally observed when the change of $\eta$ against the SIF was analyzed.

These results show that the resolution analysis provides a lower bound to the actual resolution of the open-loop system. The controller itself induces its own error, which will be assessed in the next section.

\subsection{Closed-Loop Response}

Figure 7 shows the input, output and error signals of the implemented feedback system.

From these results it is concluded that such a system is able to drive an SIF history with 
a very small error (Figure $7(\mathrm{~b})$ ) for SIF amplitudes ranging from 0.008 to $0.04 \mathrm{MPa} \sqrt{\mathrm{m}}$

(Figure $7(\mathrm{a})$ ). The errors are the highest at slope changes, as expected from the simulated system (Figure 4). Their absolute level is less than $5 \times 10^{-4} \mathrm{MPa} \sqrt{\mathrm{m}}$, i.e., one hundred times less the maximum SIF level, the SIF history was indeed controlled in a very satisfactory manner. 1437 pictures were acquired during this experiment.

On a more quantitative basis, the RMS error is equal to $8 \times 10^{-5} \mathrm{MPa} \sqrt{\mathrm{m}}$ when the whole history is analyzed. The latter includes the 11 slope changes of the SIF history (Figure $7(\mathrm{a})$ ). When these changes are not considered, the RMS error decreases by a factor of 3 to reach $2.7 \times 10^{-5} \mathrm{MPa} \sqrt{\mathrm{m}}$. This last value includes the resolution of the DIC measurements, the SIF extraction and the performance of the controller itself. When compared with the a priori SIF resolution $\left(\sigma_{K}=2 \times 10^{-6} \mathrm{MPa} \sqrt{\mathrm{m}}\right)$, and that obtained with an open-loop configuration $\left.\left(\sigma_{K}=10^{-5} \mathrm{MPa} \sqrt{\mathrm{m}}\right)\right)$, it is concluded that the controller contributes to about $60 \%$ of the total error. This level is to be expected from a standard closed-loop system.

\section{Test on Commercially Pure Titanium}

Having shown that the designed controller is able to accurately prescribe SIF histories on a soft material, the final step of the present study is to perform the same type of experiment on a stiffer material (i.e., with a Young's modulus in the 100-GPa range). In the present case, commercially pure titanium (i.e., T35) is chosen. This type of material has already been studied to estimate crack propagation parameters $[36,42]$. The sample has a CCT geometry with a width of $30 \mathrm{~mm}$, a height of $150 \mathrm{~mm}$, and thickness of $0.3 \mathrm{~mm}$. The initial crack size is equal to $6 \mathrm{~mm}$. In the present case, it is believed that the plane stress solution is a good approximation. 
Figure 8(a) shows the input and output signals for the four applied cycles when the SIF range varies between 6 and $9 \mathrm{MPa} \sqrt{\mathrm{m}}$. In the present case, 563 pictures are acquired. The overall response of the controller is deemed satisfactory as no particular deviations are observed. Contrary to the previous case, the change of slope is not accompanied by a higher error (Figure $8(\mathrm{~b})$ ). This is due to the fact that the overall level of the latter is significantly higher. The RMS error with respect to linearity is found to be equal to $9.2 \times 10^{-2} \mathrm{MPa} \sqrt{\mathrm{m}}$. Being more than 60 times lower than the minimum prescribed amplitude, the implemented system can be used to control cyclic tests with prescribed SIF histories

For the chosen (T35) grade, the Young's modulus is equal to 100 GPa and Poisson's ratio $\nu=0.33$ [36]. With these elastic parameters, the a priori SIF resolution becomes

$$
\sigma_{K}=1.4 \times 10^{-2} \mathrm{MPa} \sqrt{\mathrm{m}}
$$

since the physical size of one pixel is $p=13.4 \mu \mathrm{m}, R_{\max }=400$ pixels, and $\ell=32$ pixels. For the open-loop system, an RMS error with respect to linearity is found to be equal to $4.1 \times 10^{-2} \mathrm{MPa} \sqrt{\mathrm{m}}$ (to be compared to $9.2 \times 10^{-2} \mathrm{MPa} \sqrt{\mathrm{m}}$ for the closed-loop system). In the present case, there is a factor 3 difference between the a priori SIF resolution and the RMS linearity error of the open-loop response, and a factor 7 difference between the SIF resolution and the RMS error of the SIF controller. With the chosen SIF amplitudes whose level is close to SIF threshold in high cycle fatigue [36], it can be concluded that the controller can be used to monitor crack opening during fatigue cycles with SIF amplitudes greater than a few $\operatorname{MPa} \sqrt{\mathrm{m}}$.

Figure 9 shows the change of the measured load with respect to the prescribed SIF. There is a linear relationship between these two quantities, thereby validating the small scale yielding hypothesis. When measuring the RMS error to linearity, it is equal 
to $9.5 \times 10^{-2} \mathrm{MPa} \sqrt{\mathrm{m}}$ when expressed in terms of SIF, or $1.7 \mathrm{~N}$ in terms of load. The former is very close to the RMS error observed for the controller itself. The latter is of the order of what is expected from the used load cell (see Figure 5). This last result validates the whole procedure developed herein.

\section{Summary}

It was shown that it is possible to control in a mechanical test a stress intensity factor (SIF) history by resorting to digital images. In the present case, a local approach to digital image correlation is considered to measure the displacement field. The latter is then post-processed to evaluate the rigid body motions of the crack tip and the mode I stress intensity factor (SIF). Since this procedure requires almost 3 seconds per control cycle, a two-loop system is implemented. The inner loop is displacement-controlled and the outer loop is SIF-controlled.

Resolution analyses were performed to design the controller. A priori estimates of displacement, strain and SIF resolutions were validated a posteriori by analyzing a series of ten pictures for which no displacements were prescribed. The SIF resolution was then compared with the linearity error of the open-loop and closed-loop system. There is almost an order of magnitude difference when the former is compared with the latter ones. This result is to be expected as it includes not only measurement uncertainties but also other aspects of the controller. With the two studied cases, it was shown that the SIF range is significantly larger (i.e., almost two orders of magnitude) compared with the linearity error of the controller. It is concluded that the SIF histories prescribed herein are sufficiently accurate to monitor gradual crack openings and closures in cyclic tests. 
Having validated the implemented controller, the next step is to consider crack propagation. This step requires an accurate estimation of the crack tip position, which was not discussed herein. The same procedure as that proposed by Hamam et al. [17], which is based on canceling out the amplitude of the first supersingular displacement field, will be considered. As already mentioned, additional displacement fields accounting for, say the T-stress or other subsingular contributions $[43,36]$, may also be added. They will yield a more robust estimate of the stress intensity factor. These issues being solved, gradual propagation (e.g., in high cycle fatigue) will enable the controller to be used in a 'real time' configuration since the crack tip motion per cycle is expected to be very small so that the determination of its position will be needed only at large time increments.

\section{Acknowledgments}

This work was partially supported by a grant from Région Île-de-France (plateforme francilienne d'expérimentation mécanique de troisième génération). The authors thank Dr. Roux for useful discussions during the project. 


\section{References}

1. J. Lemaitre and J.-L. Chaboche, Mechanics of Solid Materials, (Cambridge University Press, Cambridge (UK), 1990).

2. M. F. Kanninen and C. H. Popelar, Advanced Fracture Mechanics, (Oxford University Press, Oxford (UK), 1985).

3. M. L. Williams, On the stress distribution at the base of a stationary crack, ASME J. Appl. Mech. 24 (1957) 109-114.

4. A. A. Wells, Unstable crack propagation in metals: cleavage and fast fracture, Proceedings Crack Propagation Symp., (1961), 210-230.

5. J. R. Rice, Mechanics of crack tip deformation and extension by fatigue, Proceedings Fatigue crack propagation, STP 415, (ASTM, Philadelphia (USA), 1967), 247-309.

6. G. R. Irwin, Analysis of the Stresses and Strains near the End of a Crack Traversing a Plate, ASME J. Appl. Mech. 24 (1957) 361-364.

7. A. A. Griffith, The Phenomenon of Rupture and Flow in Solids, Phil. Trans. Roy. Soc. London A221 (1920) 163-198.

8. J. D. Eshelby, The force on an elastic singularity, Phil. Trans. R. Soc. London A 244 (1951) $87-112$.

9. G. P. Cherepanov, The propagation of cracks in a continuous medium, J. Appl. Math. Mech. 31 [3] (1967) 503-512.

10. J. R. Rice, A Path Independent Integral and Approximate Analysis of Strain Concentrations by Notches and Cracks, ASME J. Appl. Mech. 35 (1968) 379-386.

11. J. Zuidema and M. Mannesse, A model for predicting slant fatigue crack growth in A1 2024, Eng. Fract. Mech. 34 (1989) 445-456.

12. J. E. Srawley and B. Gross, Stress Intensity Factors for Crackline-Loaded Edge-Crack Specimens, Materials Research and Standards 7 (1967) 155-162.

13. G. C. Pulos and W. G. Knauss, Constant stress intensity factors through closed-loop control, Int. J. Fract. 63 [2] (1993) 101-112.

14. M. A. Sutton, J.-J. Orteu and H. Schreier, Image correlation for shape, motion and deformation measurements: Basic Concepts, Theory and Applications, (Springer, New York, NY (USA), 2009). 
15. F. Hild and S. Roux, Digital Image Correlation, in: Optical Methods for Solid Mechanics. A Full-Field Approach, P. Rastogi and E. Hack, eds., (Wiley-VCH, Weinheim (Germany), $2012183-228$.

16. W. T. Riddel, R. S. Piascik, M. A. Sutton, W. Zhao, S. R. McNeill and J. D. Helm, Determining fatigue crack opening loads from near-crack-tip displacement measurements, in: Advances in fatigue crack closure measurement and analysis: second volume, STP 1343, R. C. McClung and J. Newman, J.C., eds., (ASTM, 1999), 157-174.

17. R. Hamam, F. Hild and S. Roux, Stress intensity factor gauging by digital image correlation: Application in cyclic fatigue, Strain 43 (2007) 181-192.

18. J. W. Phillips, Fracture Mechanics and Experimental Analysis, Experimental Techniques 6 [3] (1982) 11-13.

19. S. R. McNeill, W. H. Peters and M. A. Sutton, Estimation of stress intensity factor by digital image correlation, Eng. Fract. Mech. 28 [1] (1987) 101-112.

20. J. Abanto-Bueno and J. Lambros, Investigation of crack growth in functionally graded materials using digital image correlation, Eng. Fract. Mech. 69 (2002) 1695-1711.

21. S. Roux and F. Hild, Stress intensity factor measurements from digital image correlation: post-processing and integrated approaches, Int. J. Fract. 140 [1-4] (2006) 141-157.

22. S. Yoneyama, T. Ogawa and Y. Kobayashi, Evaluating mixed-mode stress intensity factors from full-field displacement fields obtained by optical methods, Eng. Fract. Mech. $\mathbf{7 4}$ (2007) 1399-1412.

23. C. G'Sell, J.-M. Hiver, A. Dahnoun and A. Souahi, Video-Controlled Tensile Testing of Polymers and Metals Beyond the Necking Point, J. Mat. Sci. 27 (1992) 5031-5039.

24. X. Fayolle, S. Calloch and F. Hild, Controlling testing machines with digital image correlation, Exp. Tech. 31 [3] (2007) 57-63.

25. X. Fayolle, Corelli ${ }^{F I C}$ : programme de pilotage d'essais asservis sur un facteur d'intensité des contraintes, (MSc. dissertation, CNAM Paris, 2008).

26. E. Durif, J. Réthoré, A. Combescure, M. Fregonese and P. Chaudet, Controlling Stress Intensity Factors During a Fatigue Crack Propagation Using Digital Image Correlation and a Load Shedding Procedure, Exp. Mech. 52 (2012) 1021-1031.

27. ISO, International Vocabulary of Basic and General Terms in Metrology (VIM), (International Organization for Standardization, Geneva (Switzerland), 1993). 
28. ISO, Guide to the Expression of Uncertainty in Measurements (GUM), (International Organization for Standardization, Geneva (Switzerland), 1995).

29. D. J. Chen, F. P. Chiang, Y. S. Tan and H. S. Don, Digital Speckle-Displacement Measurement Using a Complex Spectrum Method, Appl. Opt. 32 (1993) 1839-1849.

30. Y. Berthaud, J. Scholz and J. Thesing, Méthodes optiques et acoustiques de mesures des caractéristiques mécaniques, Proc. Colloque national MECAMAT "Mécanismes et mécanique des grandes déformations" (1997), 77-80.

31. F. P. Chiang, Q. Wang and F. Lehman, New Developments in Full-Field Strain Measurements Using Speckles, in: Non-Traditional Methods of Sensing Stress, Strain and Damage in Materials and Structures (ASTM, Philadelphia (USA), 1997), STP 1318 156-169.

32. L. Chevalier, S. Calloch, F. Hild and Y. Marco, Digital Image Correlation used to Analyze the Multiaxial Behavior of Rubber-Like Materials, Eur. J. Mech. A/Solids 20 (2001) 169-187.

33. F. Hild and S. Roux, Comparison of local and global approaches to digital image correlation, Exp. Mech. 52 [9] (2012) 1503-1519.

34. S. Bergonnier, F. Hild and S. Roux, Digital image correlation used for mechanical tests on crimped glass wool samples, J. Strain Analysis 40 [2] (2005) 185-197.

35. J. Réthoré, S. Roux and F. Hild, Noise-robust Stress Intensity Factor Determination from Kinematic Field Measurements, Eng. Fract. Mech. 75 [13] (2008) 3763-3781.

36. F. Mathieu, F. Hild and S. Roux, Identification of a crack propagation law by digital image correlation, Int. J. Fat. 36 (2012) 146-154.

37. H. Leclerc, J.-N. Périé, S. Roux and F. Hild, Integrated digital image correlation for the identification of mechanical properties, in: MIRAGE 2009, A. Gagalowicz and W. Philips, eds., (Springer, Berlin, 2009), LNCS 5496 161-171.

38. Mathworks, Simulation and Model-Based Simulation, version R2012b, (the MathWorks, inc. (http://www.mathworks.com), 2012).

39. H. Tada, P. C. Paris and G. R. Irwin, The Stress Analysis of Cracks Handbook, (Del Research, St Louis (USA), 1985).

40. National Instrument, LabVIE $W^{T M}$, user's manual (http://www.ni.com), 2012).

41. Ward IM, Hadley DW (1993) An introduction to the mechanical properties of solid polymers. John Wiley and Sons, New York (USA). 
42. F. Mathieu, F. Hild and S. Roux, Image-based Identification procedure of a crack propagation law, Eng. Fract. Mech. 103 (2013) 48-59.

43. C. Henninger, S. Roux and F. Hild, Enriched kinematic fields of cracked structures, Int. J. Solids Struct. 47 (2010) 3305-3316. 


\section{List of Figures}

1 Standard displacement resolution $\sigma_{u}$ as a function of the ZOI size $\ell$. The symbols correspond to the analysis with the reference picture of the SIF-controlled test on the studied elastomer. The solid line is the interpolation given by Equation $(? ?) \ldots \ldots \ldots . \ldots . \ldots 24$

2 Dimensionless standard SIF resolution $\sigma_{K} \sqrt{n_{x} n_{y} R_{\max }} / E \sigma_{u}$ as a function of the Poisson's ratio $\nu$ for different numbers $n_{x} n_{y}$ of ZOIs. The symbols correspond to the value of the Poisson's ratio of the studied elastomer, and the solid line to the approximation given by Equation (??) 25

3 Block Diagram of the two-loop controller. Points R, E and O correspond to the signals shown in Figure $4 \ldots \ldots \ldots 26$

4 Simulated response of the controller (see Figure 3). (a) Reference (R) SIF history, $(\mathrm{b})$ error signal $(\mathrm{E}=\mathrm{O}-\mathrm{R}) \ldots \ldots 27$

5 Stress / strain response of a tensile test on the studied elastomer. The stress fluctuations are due to the standard resolution of the load cell (i.e., of the order of $1 \mathrm{~N}$ or $0.02 \mathrm{MPa}) \ldots \ldots \ldots$

6 Analysis of the controller as an open-loop system. Change of the SIF as a function of the picture number when two loading/unloading cycles are

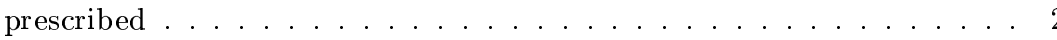

$7 \quad$ Analysis of the controller as a closed-loop system. (a) Comparison of the input and output SIF signals of the controller when a single edge cracked sample made of elastomer is tested. (b) Error signal in the controller . . 30 
8 (a) Comparison of the input and output SIF signals of the controller when a CCT sample made of T35 is tested. (b) Corresponding error signal in the controller ................... 31

9 Measured load vs. SIF for the CCT sample made of T35. The crosses are experimental points and the solid line corresponds to a linear interpolation to be expected when the small scale yield hypothesis applies . . 


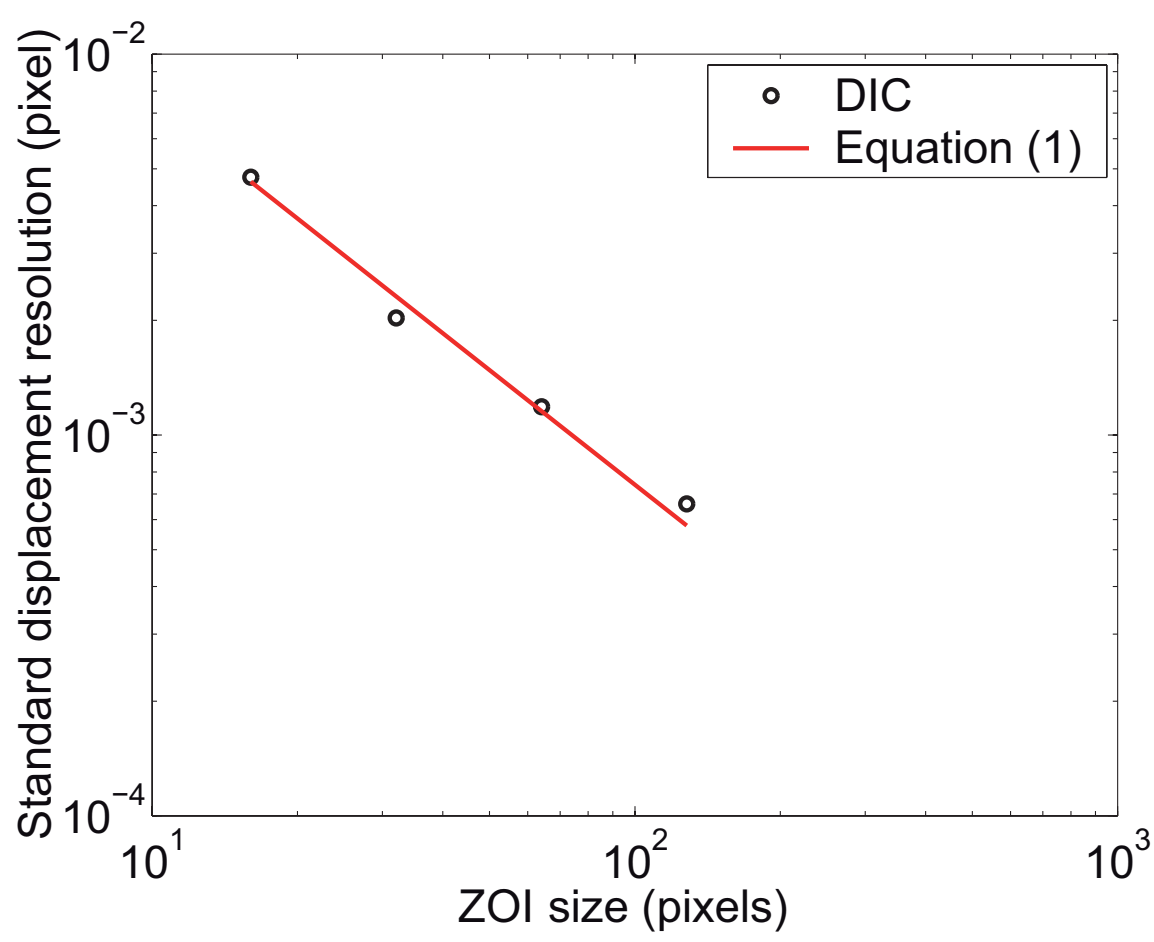

Fig. 1 Standard displacement resolution $\sigma_{u}$ as a function of the ZOI size $\ell$. The symbols correspond to the analysis with the reference picture of the SIF-controlled test on the studied elastomer. The solid line is the interpolation given by Equation (1) 


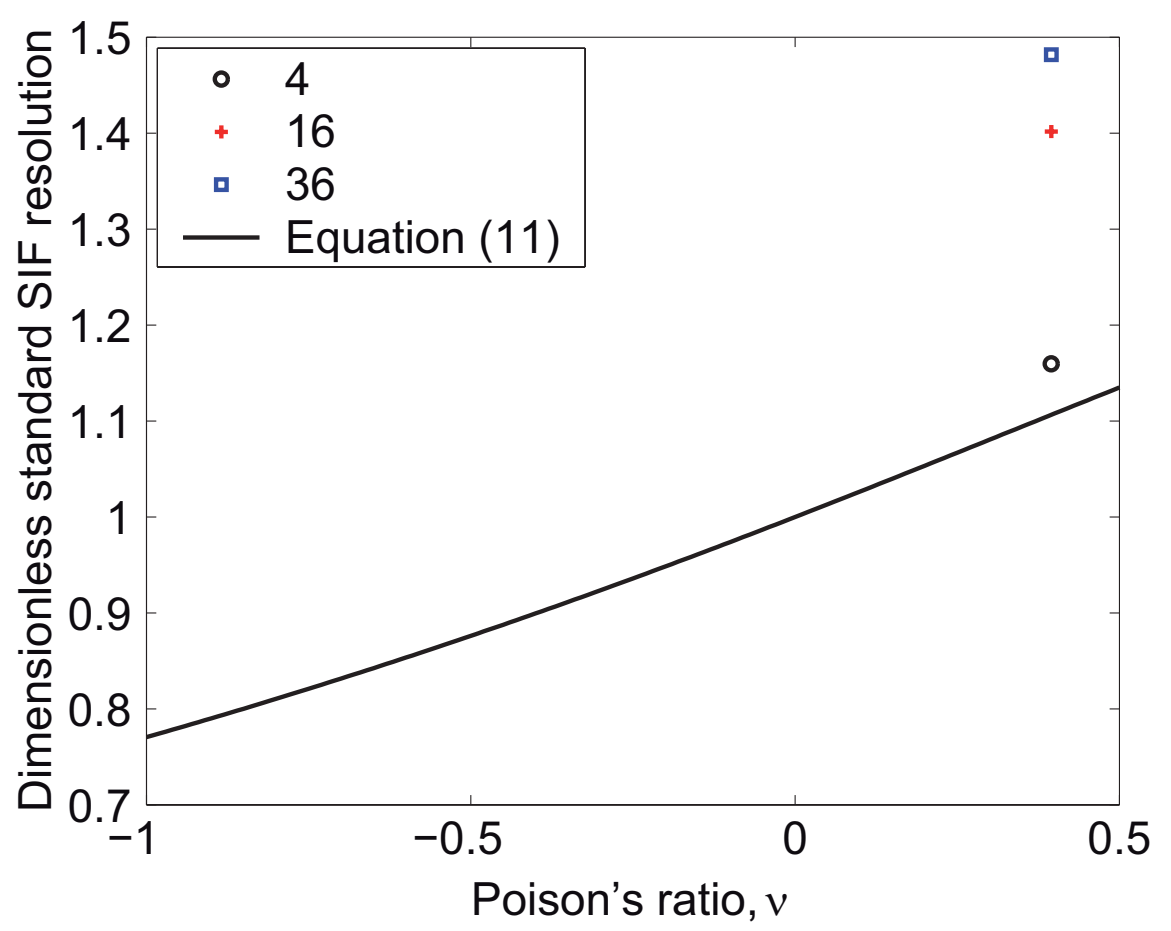

Fig. 2 Dimensionless standard SIF resolution $\sigma_{K} \sqrt{n_{x} n_{y} R_{\max }} / E \sigma_{u}$ as a function of the Poisson's ratio $\nu$ for different numbers $n_{x} n_{y}$ of ZOIs. The symbols correspond to the value of the Poisson's ratio of the studied elastomer, and the solid line to the approximation given by Equation (11) 


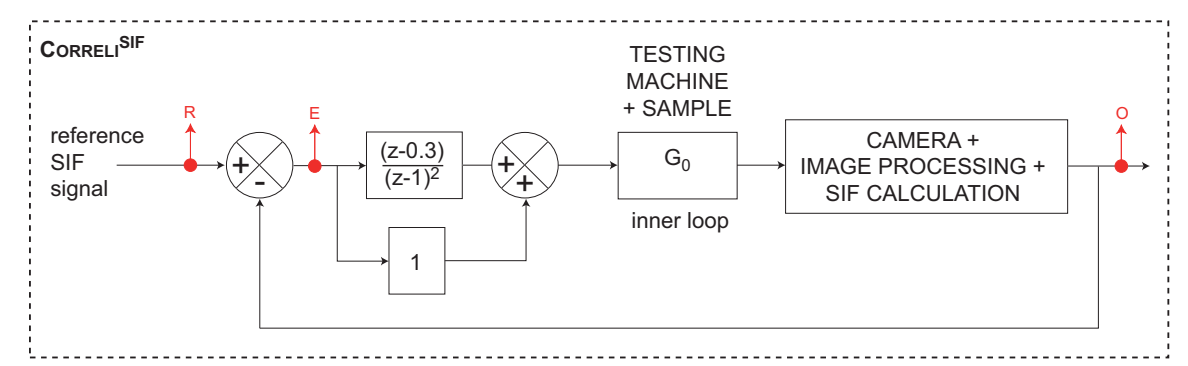

Fig. 3 Block Diagram of the two-loop controller. Points R, E and O correspond to the signals shown in Figure 4 


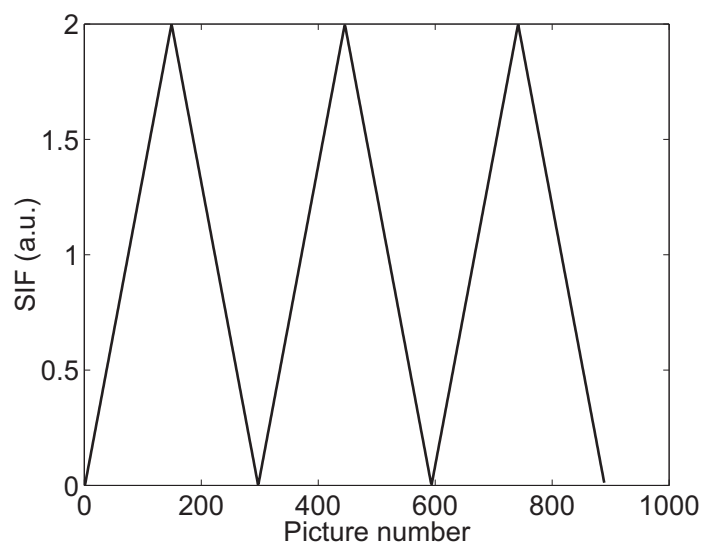

(a)

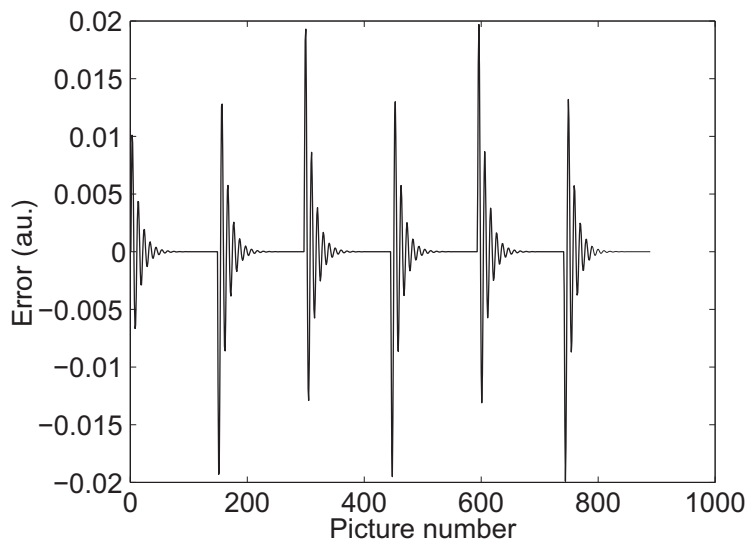

(b)

Fig. 4 Simulated response of the controller (see Figure 3). (a) Reference (R) SIF history, (b) error signal $(\mathrm{E}=\mathrm{O}-\mathrm{R})$ 


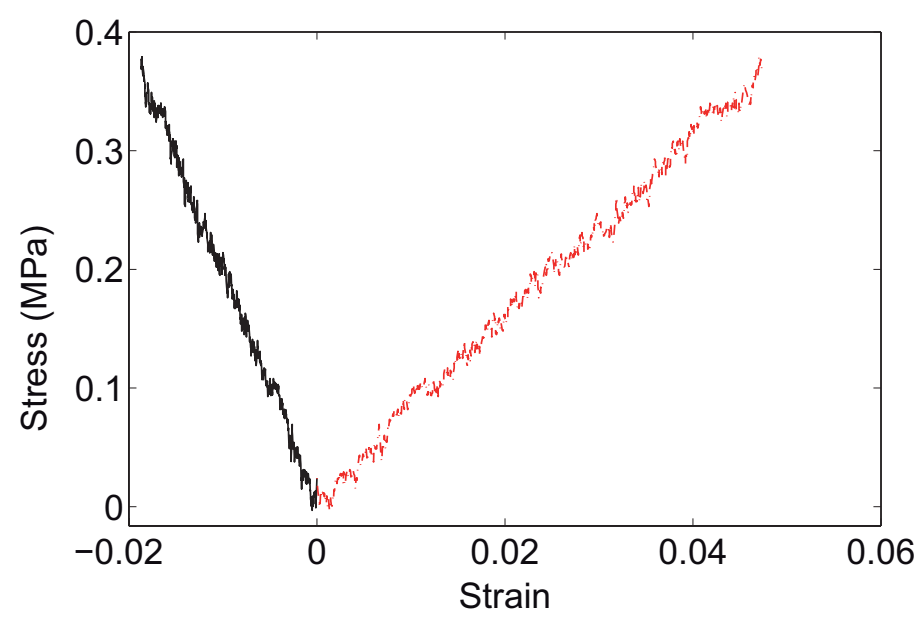

Fig. 5 Stress / strain response of a tensile test on the studied elastomer. The stress fluctuations are due to the standard resolution of the load cell (i.e., of the order of $1 \mathrm{~N}$ or $0.02 \mathrm{MPa}$ ) 


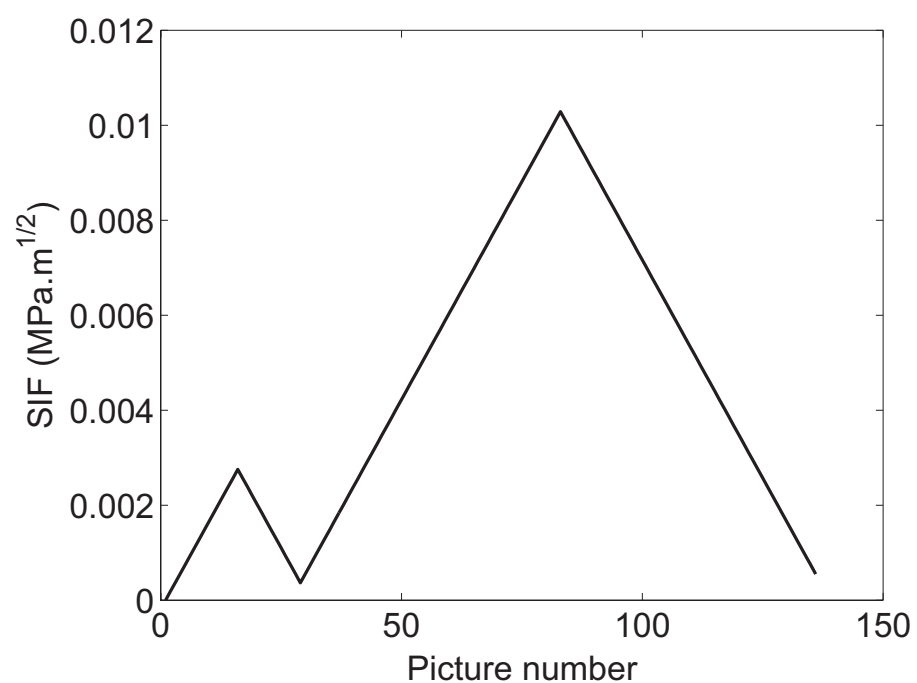

Fig. 6 Analysis of the controller as an open-loop system. Change of the SIF as a function of the picture number when two loading/unloading cycles are prescribed 


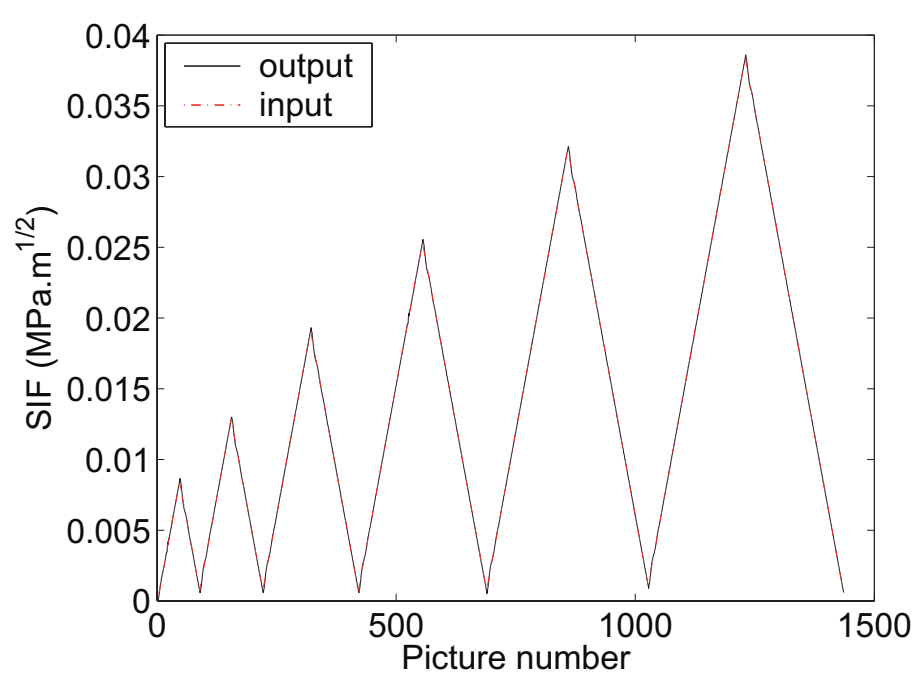

(a)

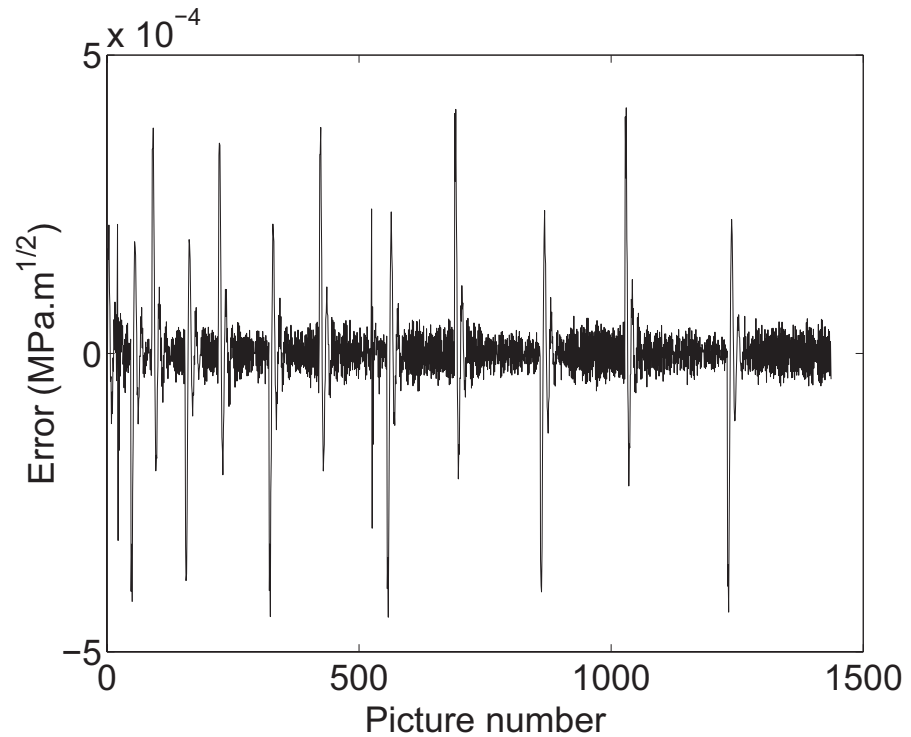

(b)

Fig. 7 Analysis of the controller as a closed-loop system. (a) Comparison of the input and output SIF signals of the controller when a single edge cracked sample made of elastomer is tested. (b) Error signal in the controller 


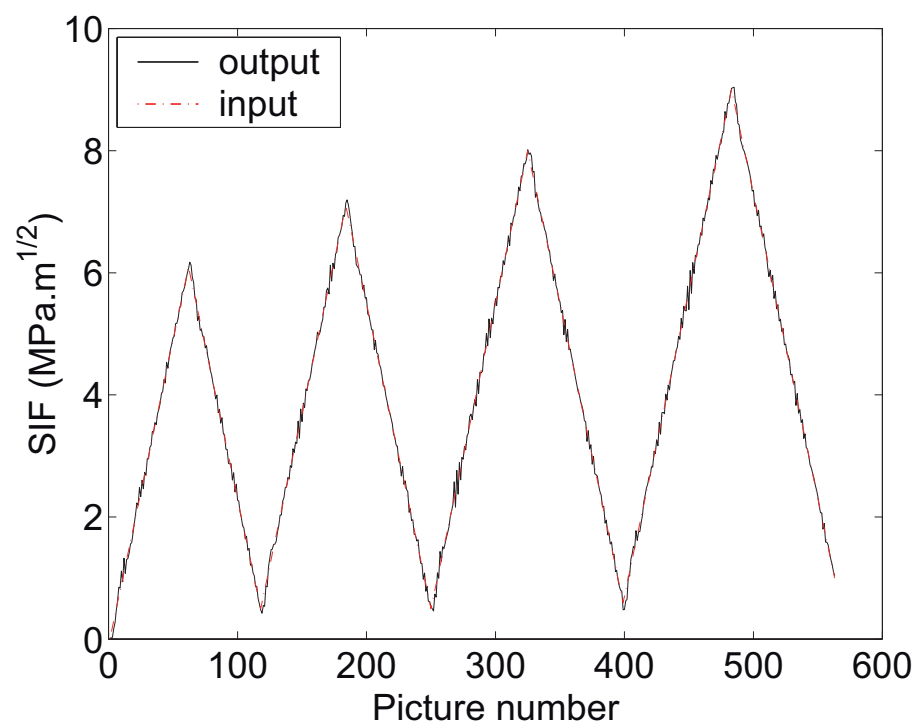

(a)

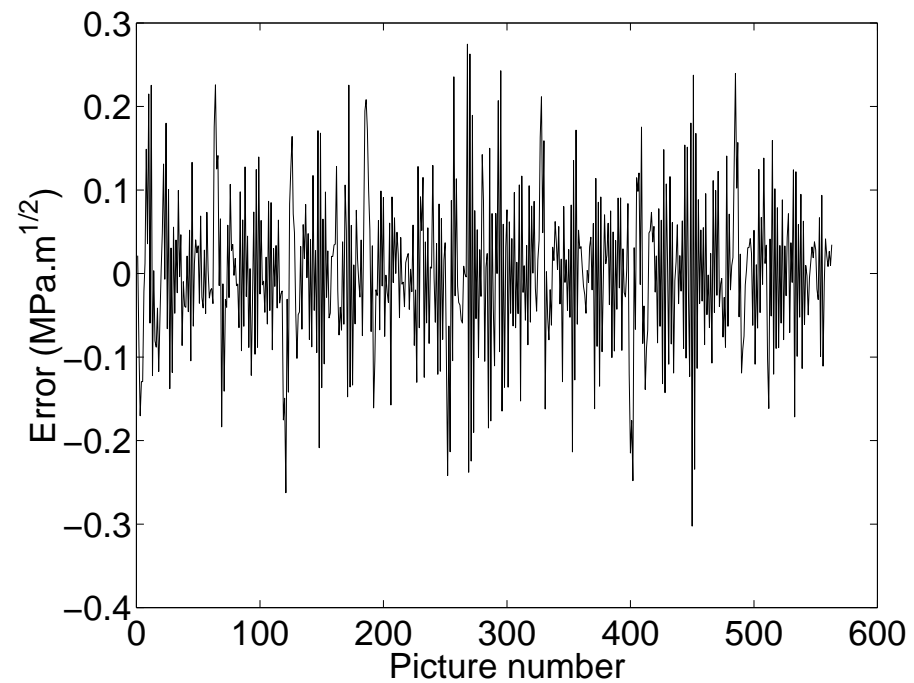

(b)

Fig. 8 (a) Comparison of the input and output SIF signals of the controller when a CCT sample made of T35 is tested. (b) Corresponding error signal in the controller 


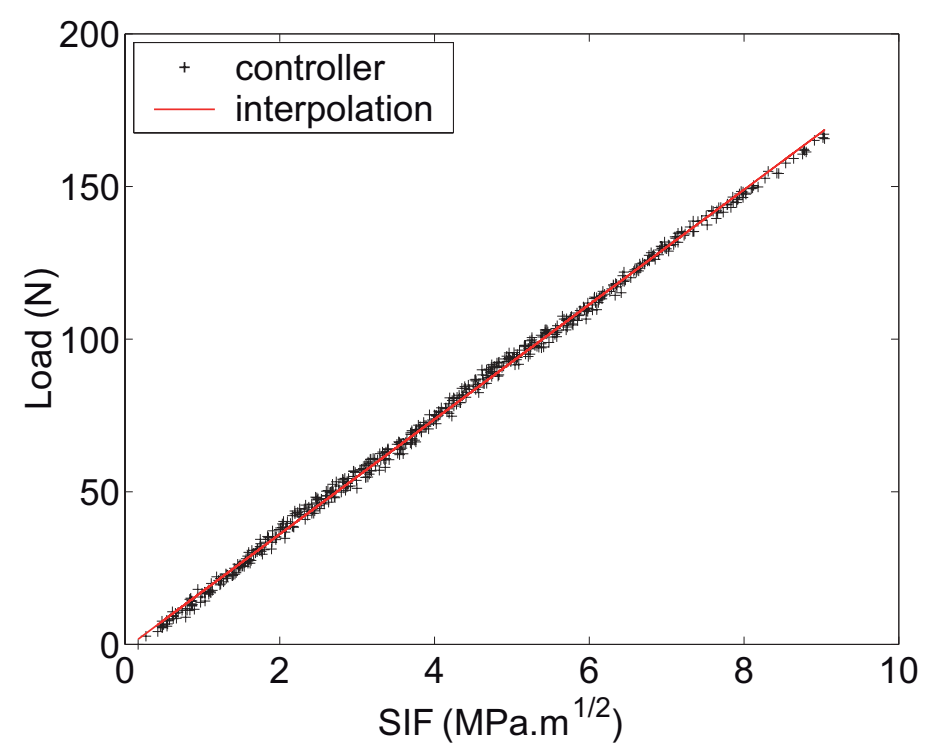

Fig. 9 Measured load vs. SIF for the CCT sample made of T35. The crosses are experimental points and the solid line corresponds to a linear interpolation to be expected when the small scale yield hypothesis applies 